\title{
Differential resistance of human embryonic stem cells and somatic cell types to hydrogen peroxide-induced genotoxicity may be dependent on innate basal intracellular ROS levels
}

\author{
Kumar Jayaseelan Vinoth ${ }^{1,2}$, Jayapal Manikandan ${ }^{3,4}$, Swaminathan Sethu $^{4}$, \\ Lakshmidevi Balakrishnan ${ }^{4}$, Alexis Heng ${ }^{5,6}, \mathrm{Kai} \mathrm{Lu}^{7,8}$, Anuradha Poonepalli, \\ Manoor Prakash Hande ${ }^{4,9,10}$, Tong Cao ${ }^{1,10,11}$
}

${ }^{1}$ Stem Cell Laboratory, Faculty of Dentistry, National University of Singapore, Singapore

${ }^{2}$ Department of Oral \& Maxillofacial Surgery, National Dental Centre, SingHealth, Singapore

${ }^{3}$ School of Anatomy, Physiology and Human Biology, the University of Western Australia, Crawley, Australia

${ }^{4}$ Department of Physiology, Yong Loo Lin School of Medicine, National University of Singapore, Singapore

${ }^{5}$ Department of Biological Sciences, Faculty of Science \& Technology, Sunway University, Selangor Darul Ehsan, Malaysia

${ }^{6}$ Department of Biosystems Science \& Engineering, ETH-Zurich, Basel, Switzerland

${ }^{7}$ Institute for Integrated Cell-Material Sciences, Kyoto University, Japan

${ }^{8}$ Faculty of Health Sciences, University of Macau, Macau, China

${ }^{9}$ Tembusu College, University Town, National University of Singapore, Singapore

${ }^{10}$ National University of Singapore Graduate School for Integrative Sciences and Engineering (NGS), Singapore

${ }^{11}$ National University of Singapore Tissue Engineering Program (NUSTEP), Life Sciences Institute, National University of Singapore, Singapore

\footnotetext{
Abstract

Previously, we demonstrated that undifferentiated human embryonic stem cells (hESC) displayed higher resistance to oxidative and genotoxic stress compared to somatic cells, but did not further probe the underlying mechanisms. Using $\mathrm{H}_{2} \mathrm{O}_{2}$-induced genotoxicity as a model, this study investigated whether higher resistance of $\mathrm{hESC}$ to oxidative and genotoxic stress could be due to lower innate basal intracellular levels of reactive oxygen species (ROS), as compared to their differentiated fibroblastic progenies (H1F) and two other somatic cell types - human embryonic palatal mesenchymal (HEPM) cells and peripheral blood lymphocytes (PBL). Comet assay demonstrated that undifferentiated hESC consistently sustained lower levels of DNA damage upon acute exposure to $\mathrm{H}_{2} \mathrm{O}_{2}$ for $30 \mathrm{~min}$, compared to somatic cells. DCFDA and HE staining with flow cytometry showed
}

Correspondence address: Assoc. Prof. T. Cao

Faculty of Dentistry, National University of Singapore

11 Lower Kent Ridge Road, Singapore 119083

tel.: +65-6772 6845 (Office), fax: +65-6778 5742

e-mail: dencaot@nus.edu.sg 
that undifferentiated hESC had lower innate basal intracellular levels of reactive oxygen species compared to somatic cells, which could lead to their higher resistance to genotoxic stress upon acute exposure to $\mathrm{H}_{2} \mathrm{O}_{2}$. (Folia Histochemica et Cytobiologica 2015, Vol. 53, No. 2, 169-174)

Key words: human ESC; genotoxicity; $\mathrm{H}_{2} \mathrm{O}_{2}$; reactive oxygen species; comet assay; flow cytometry

\section{Introduction}

Oxidative stress and genotoxicity are known to be closely intertwined, with abundant evidence in the scientific literatures linking oxidative stress to DNA damage and mutations [1,2]. Moreover, several toxicological studies have conclusively demonstrated that many genotoxic agents in fact cause an elevation in intracellular levels of reactive oxygen species (ROS), which in turn contribute to DNA damage [3, 4]. Our previous studies have shown that undifferentiated human embryonic stem cells (hESC) displayed higher resistance to both oxidative and genotoxic stress compared to somatic cell types $[5,6]$, but the underlying molecular mechanisms for this observation have not been well documented.

The aim of the study, based on $\mathrm{H}_{2} \mathrm{O}_{2}$-induced genotoxicity as a model system, was to investigate whether the observed higher resistance of undifferentiated hESC to oxidative and genotoxic stress could be due to lower innate basal intracellular levels of reactive oxygen species within undifferentiated hESC, as compared to somatic cell types. Three different somatic cell types were examined in this study - human embryonic palatal mesenchymal (HEPM) cells, peripheral blood lymphocytes (PBL), and differentiated fibroblastic progenies (H1F) of hESC.

\section{Material and methods}

Human embryonic stem cell culture. Undifferentiated hESC of the H1 line (Wicell Inc., Madison, WI, USA) were cultured on feeder layers of mitotically-inactivated mouse embryonic fibroblasts (MEF) in DMEM-F12 medium supplemented with $20 \%(\mathrm{v} / \mathrm{v})$ knockout serum replacement, $1 \mathrm{mM}$ glutamine, $4 \mathrm{ng} / \mathrm{mL}$ basic fibroblast growth factor (bFGF), $1 \%$ (v/v) non-essential amino acids (all purchased from Gibco BRL Inc., Grand Island, NY, USA), and $0.1 \mathrm{mM}$ $\beta$-mercaptoethanol (DMSO, SigmaAldrich, St Louis, MO, USA), within a humidified $5 \% \mathrm{CO}_{2}$ incubator at $37^{\circ} \mathrm{C}$. The hESC cultures were serially passaged every 5-7 days at a split ratio of $1: 6$, through treatment with $1 \mathrm{mg} / \mathrm{mL}$ collagenase type IV (Gibco) followed by manual dissociation of the hESC colonies by pipetting. The resulting hESC clumps were re-plated on fresh MEF feeder layers seeded on culture plates pre-coated with $0.1 \%$ porcine gelatine (Gibco). For microarray analysis that required a pure population of hESCs without contaminating MEFs, the hESCs were subjected to 2 passages under feeder-free conditions with Matrigel (BD Bioscience, Franklin Lakes, NJ, USA), before being utilized for experiments.

Differentiation of human embryonic stem cells into fibroblastic progenies (H1F). Dissociated hESC clumps were initially seeded in T-75 flasks that were pre-coated with $0.1 \%$ porcine gelatine, and were induced to differentiate into fibroblasts by culturing in Dulbecco's Modified Essential Medium (DMEM; NUMI Media Preparation Facility NUS, Singapore) supplemented with $10 \%(\mathrm{v} / \mathrm{v})$ fetal bovine serum (FBS; Hyclone, USA), within a humidified $5 \% \mathrm{CO}_{2}$ incubator. Subsequent passages of cells were plated directly in T-75 flasks without the gelatine coating. The differentiated fibroblastic progenies obtained were referred to as $\mathrm{H} 1 \mathrm{hESC}$ derived progenies (H1F). Cells were cultured to the $5^{\text {th }}$ passage, before being utilized in experiments.

Culture of human embryonic palatal mesenchymal cells and peripheral blood lymphocytes. To isolate human peripheral blood lymphocytes, heparinized venous blood was subjected to Ficoll-Hypaque (GE Healthcare Bio-Sciences, Singapore) density gradient centrifugation at $500 \mathrm{~g}$, without using brakes for $20 \mathrm{~min}$ at room temperature. After collection of the lymphocyte layer above the Ficoll gradient, the cells were washed three times in phosphate-buffered saline (PBS), followed by cell counting with a hemocytometer. The culture milieu utilized for cultures of human peripheral lymphocytes was Roswell Park Memorial Institute (RPMI) 1640 medium (Gibco) supplemented with $10 \%$ (v/v) FBS (Hyclone) and 1\% (v/v) Penicillin/Streptomycin (Gibco).

Human embryonic palatal mesenchymal cells (HEPM, ATCC Catalogue no: CRL1486) were cultured in DMEM (NUMI Media Preparation Facility, Singapore) supplemented with $10 \%$ (v/v) FBS (Hyclone, South Logan, UT, USA). All cell cultures were carried out within a humidified $5 \% \mathrm{CO}_{2}$ incubator at $37^{\circ} \mathrm{C}$.

Exposure of cells to hydrogen peroxide. Stock solutions of hydrogen peroxide $\left(\mathrm{H}_{2} \mathrm{O}_{2}\right.$; Kanto Chemical Co. Inc., Japan) were freshly prepared in PBS before each experiment. The four different cell types (hESC, H1F, PBL \& HEPM) were then exposed to doses of $50 \mu \mathrm{M}, 100 \mu \mathrm{M}$ and $200 \mu \mathrm{M} \mathrm{H}_{2} \mathrm{O}_{2}$ for $30 \mathrm{~min}$, and were subjected to analyses by comet assay and DCFDA/HE flow cytometry. 
Alkaline single cell gel electrophoresis assay (Comet Assay). Comet assay was utilized to quantify the extent of DNA damage. Alkaline conditions at $\mathrm{pH}$ greater than 13 allow for the detection of single stranded, double-stranded breaks, apurinic and apyridiminic sites as well as alkali adducts. Cells were lysed and then denatured under alkaline conditions to unwind the DNA and hydrolyze the damaged sites. The cell lysate was then subjected to electrophoresis. Electrophoresis induced the cleaved DNA fragments to migrate further under the electric field, as compared to undamaged DNA that remained in the nucleoidal shape. After treatment, cells were harvested and washed in PBS, followed by suspension in Hank's Balanced Salt Solution (HBSS; Gibco) supplemented with $10 \%(\mathrm{v} / \mathrm{v})$ DMSO (SigmaAldrich) and $0.5 \mathrm{M}$ ethylenediaminetetraacetic acid (EDTA, SigmaAldrich). Ten percent of the cell suspension volume was suspended in liquid agarose (Trevigen, Gaithersburg, MD, USA) and $50 \mu \mathrm{l}$ of this suspension was added to the comet assay slides (Trevigen). After solidification of the agarose at $4^{\circ} \mathrm{C}$, the slides were then left in lysis solution (Trevigen) overnight at $4^{\circ} \mathrm{C}$. Denaturation of the slides was carried out in the dark for $40 \mathrm{~min}$, in chilled alkaline electrophoresis buffer (pH 13.0-13.7) consisting of $24 \mathrm{~g}$ sodium hydroxide pellets (Merck Chemicals, Singapore), 0.5 M EDTA and distilled water. Subsequently, electrophoresis was carried at $25 \mathrm{~V}$ for $25 \mathrm{~min}$. The slides were dried and then stained with SYBR green dye (Molecular Probes, Eugene, OR, USA) prior to being observed under a Zeiss Axioplan 2 imaging fluorescence microscope (Carl Zeiss, Wetzlar, Germany) equipped with triple band filter. Images were captured using Comet Analysis Software (Metasystems, Altlussheim, Germany). Fifty comets per sample were analyzed to obtain the mean tail DNA. All measurements were performed in duplicates.

Flow cytometry with DCFDA- and hydroethidine-stained cells. For DCFDA (dichlorofluorescin diacetate) staining, cells were washed with in PBS and exposed to $5 \mu \mathrm{M}$ 5-(and-6)-chloromethyl-2,7-dichlorofluorescin diacetate

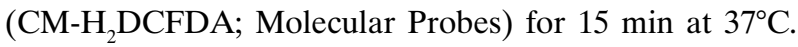
Subsequently, the cells were washed again in PBS and analyzed by flow cytometry (Coulter EPICS Elite ESP; Beckman Coulter, Fullerton, CA, USA) utilizing excitation/emission wavelengths of $488 \mathrm{~nm} / 525 \mathrm{~nm}$ respectively. At least 10,000 events were analyzed using the WinMDI (Windows multiple document interface for flow cytometry) software (Beckman Coulter Inc., Sunnyvale, CA).

For hydroethidine (HE) staining, we utilized MitoSox Red (Invitrogen, Carlsbad, CA, USA), a live-cell permeable dye with hydroethidine covalently linked to a triphosphonium cation through a hexyl carbon chain. The dye exhibits red fluorescence once it is oxidized by superoxide. Cells were incubated with MitoSox Red for $15 \mathrm{~min}$, and then were washed twice with PBS, prior to being analyzed with an Epic Profile flow cytometer using an excitation wavelength of
$510 \mathrm{~nm}$ and emission wavelength of $580 \mathrm{~nm}$. Data were analyzed for at least 10,000 events using the WinMDI software.

Statistical analysis. Statistical analysis of the experimental data was carried out with Student's $t$ test, utilizing Microsoft Excel 2003 (Microsoft Corporation, Richmond, WA, USA). The threshold of statistical significance was set at $p<0.05$.

\section{Results}

\section{DNA damage induced by hydrogen peroxide in the studied cell lines}

Normal undamaged nuclei formed a nucleoidal shape whereas damaged nuclei formed a characteristic comet-like shape when subjected to electrophoresis (Figure 1A and B). The four studied cell types were exposed to an acute dose of $\mathrm{H}_{2} \mathrm{O}_{2}$ for $30 \mathrm{~min}$ and the level of DNA damage was quantified with the comet assay. It was observed that hESCs consistently sustained lower levels of DNA damage upon acute exposure to different dosages of $\mathrm{H}_{2} \mathrm{O}_{2}$ for $30 \mathrm{~min}$, as compared to the three other cell types (Figure 1C). It was only at $200 \mu \mathrm{M}$ of $\mathrm{H}_{2} \mathrm{O}_{2}$ that there was a significant change in the tail moment of the comet assay for hESCs, with no significant differences being observed at 50 and $100 \mu \mathrm{M}$ of $\mathrm{H}_{2} \mathrm{O}_{2}$. In the case of $\mathrm{H} 1 \mathrm{~F}$ cells, significant changes in the tail moment of the comet assay were observed at $100 \mu \mathrm{M}$ and $200 \mu \mathrm{M} \mathrm{H}_{2} \mathrm{O}_{2}$, but not at $50 \mu \mathrm{M} \mathrm{H}_{2} \mathrm{O}_{2}$ concentration. By contrast, HEPM and PBL displayed significant changes in the tail moment at all three concentrations of $\mathrm{H}_{2} \mathrm{O}_{2}$ (Figure 1C).

\section{Intracellular $\mathrm{H}_{2} \mathrm{O}_{2}$ and superoxide levels as assessed by flow cytometry of DCFDA- and $\mathrm{HE}$-stained cells}

It was observed that hESCs had the lowest basal intracellular $\mathrm{H}_{2} \mathrm{O}_{2}$ levels (Figure $2 \mathrm{~A}$ ), followed by similar $\mathrm{H}_{2} \mathrm{O}_{2}$ levels in PBL and $\mathrm{H} 1 \mathrm{~F}$ (Figure 2A). Of the four cell types studied, HEPM cells had the highest basal level of $\mathrm{H}_{2} \mathrm{O}_{2}$ (Figure 2A). Similar trends in $\mathrm{O}^{2-}$ levels were observed (Figure 2B). hESCs were found to have the lowest basal intracellular $\mathrm{O}^{2-}$ levels (Figure 2B). PBL and H1F cells had similar basal levels of $\mathrm{O}^{2-}$ (Figure 2B). Again, of the 4 cell types, HEPM cells had the highest basal levels of $\mathrm{O}^{2-}$ (Figure $2 \mathrm{~B}$ ).

\section{Discussion}

Alkaline single-cell gel electrophoresis (comet assay) is a sensitive indicator of DNA damage incurred. The comet assay was carried out under alkaline conditions ( $\mathrm{pH}>13$ ), to detect all types of DNA damage, including double-strand breaks, single-strand breaks, and 


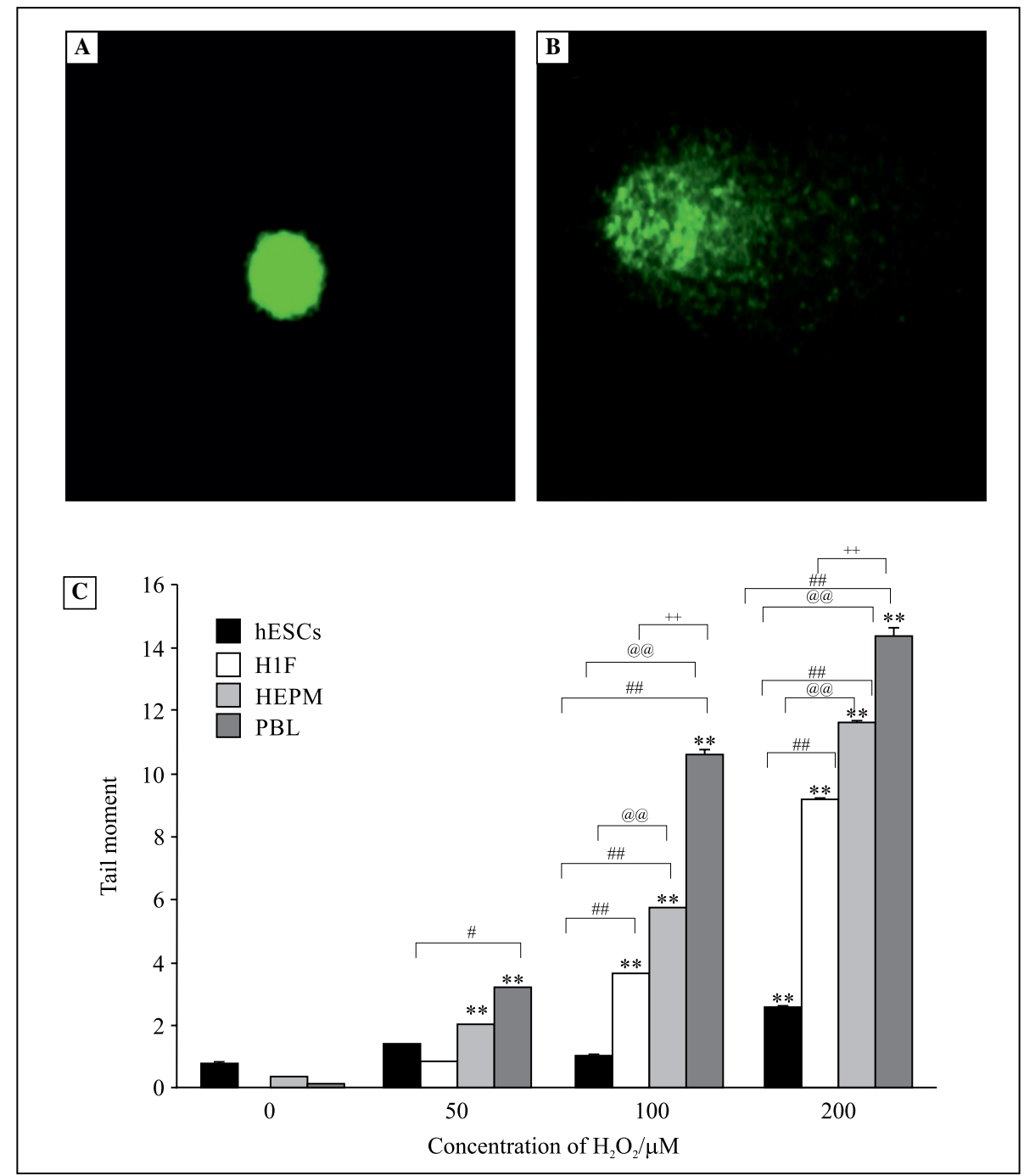

Figure 1. Extent of DNA damage of hESC, HEPM, and H1F cells as well as PBL following $\mathrm{H}_{2} \mathrm{O}_{2}$ treatment as assessed by comet assay. Representative images of (A) SYBR Green-stained undamaged nuclei of PBL cells and (B) damaged nuclei of PBL cells with characteristic comet-like shape. (C) X-axis: hESC, HEPM, PBL and H1F cells following $\mathrm{H}_{2} \mathrm{O}_{2}$ treatment. hESC (black bars), H1F (white bars), HEPM (horizontal bars) and PBL (vertical bars) were treated with $50 \mu \mathrm{M}, 100 \mu \mathrm{M}$ or $200 \mu \mathrm{M}$ of $\mathrm{H}_{2} \mathrm{O}_{2}$ for $30 \mathrm{~min}$. Y-axis: tail moment (product of tail length and fraction of DNA) corresponds to the extent of acute DNA damage as measured by the comet assay. Data represent mean value and error bars indicate standard error. Two sets of 50 comets per sample were analyzed. Abbreviations of cell type names as for Figure 1. Cell symbols: hESC — human embryonic stem cells; HEPM — human embryonic palatal mesenchymal cells; $\mathrm{H} 1 \mathrm{~F}$ - differentiated fibroblastic progenies cells; PBL - peripheral blood lymphocytes. ${ }^{*, * *} \mathrm{p}<0.05$ and $\mathrm{p}<0.01$, respectively, as compared to control (no $\mathrm{H}_{2} \mathrm{O}_{2}$ treatment) cells of the same cell type; \#, \#\# $\mathrm{p}<0.05$ and $\mathrm{p}<0.01$, respectively, as compared to $\mathrm{H}_{2} \mathrm{O}_{2}$-treated hESC cells; ${ }^{@}{ }^{@} \mathrm{p}<0.01$ as compared to $\mathrm{H}_{2} \mathrm{O}_{2}$-treated $\mathrm{H} 1 \mathrm{~F}$ cells; ${ }^{+,+} \mathrm{p}<0.05$ and $\mathrm{p}<0.01$, respectively, as compared to $\mathrm{H}_{2} \mathrm{O}_{2}$-treated HEPM cells (Student's $t$-test)

alkali labile sites [7, 8]. Consistent with our previous studies, the results of the comet assay showed that hESCs exhibited the lowest level of DNA damage upon acute exposure to $\mathrm{H}_{2} \mathrm{O}_{2}$, as compared to the three somatic cell types. We wanted to determine if the lower levels of genotoxicity observed in hESCs could be due to their lower innate basal intracellular ROS levels, as compared to somatic cells. Indeed, oxygen metabolism itself has been demonstrated to contribute to increased levels of double strand DNA breaks [9].

To evaluate whether the increased levels of DNA damage observed under comet assay were a product of increased intracellular ROS levels, flow cytometry with DCFDA and hydroxyethidine staining was applied to elucidate innate intracellular hydrogen 


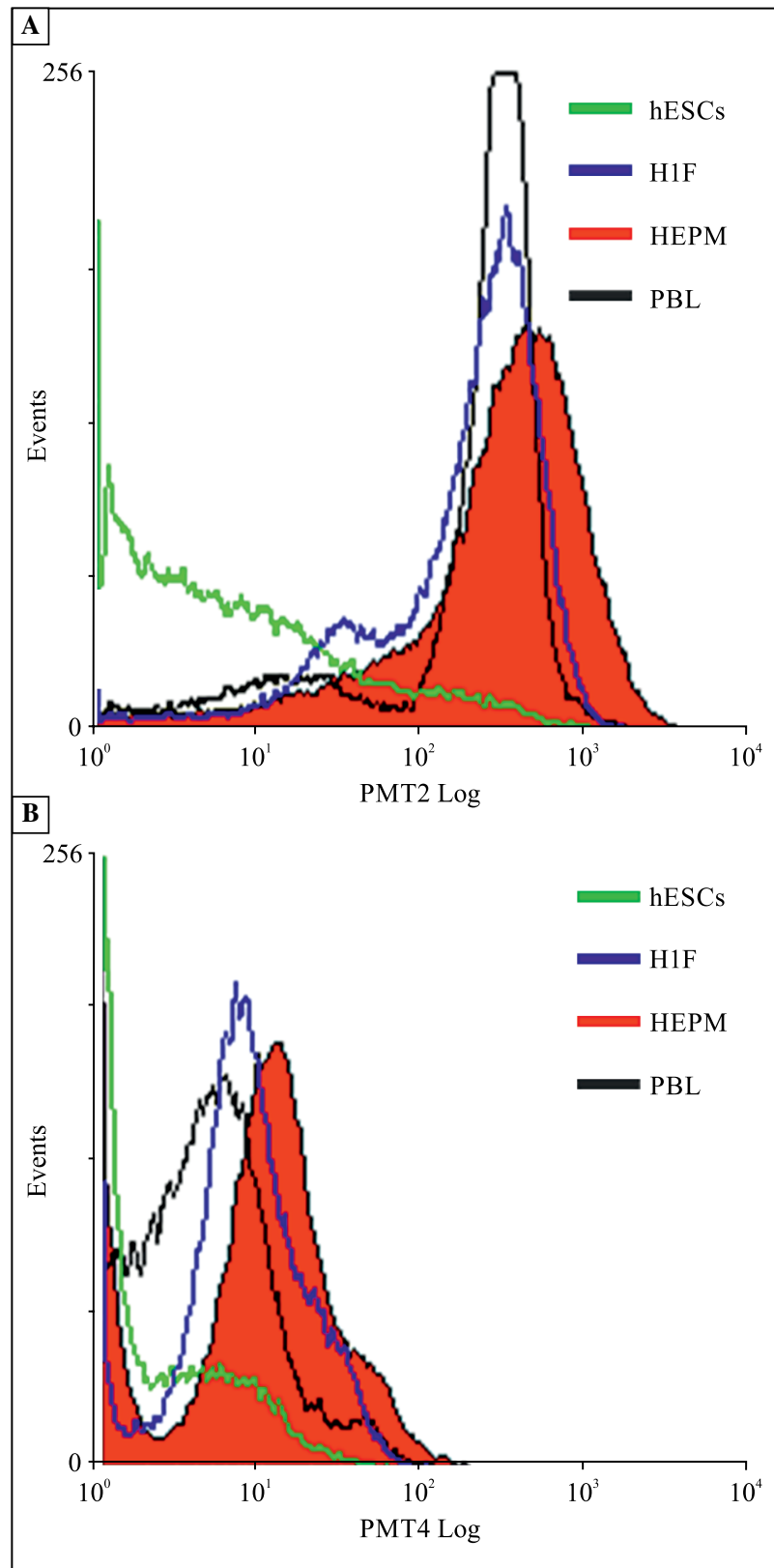

Figure 2. Endogenous hydrogen peroxide (A) and superoxide (B) levels in the studied cell types. hESC, H1F, HEPM and PBLs were treated with $100 \mu \mathrm{M}$ hydrogen peroxide for $30 \mathrm{~min}$. Then the cells were analyzed with flow cytometry for intracellular $\mathrm{H}_{2} \mathrm{O}_{2}$ formation by DCFDA staining and intracellular superoxide levels by hydroxyethidine stainin as described in Material and methods. hESCs (Green), H1F (Blue), HEPM (Red) and PBLs (Black). Fifty thousand cells were analyzed per run. Figures are representative of 3 replicate experiments. The $\mathrm{X}$-axes of the graphs represent light signal being detected by a photomultiplier tube (PMT) that is converted via a pre-amplifier to an electrical voltage output signal that is proportional to the original fluorescence intensity. This data is then subjected to log transformation at either the base 2 (PMT2 Log) or base 4 (PMT4 Log) peroxide and superoxide levels. A higher ROS level is indicated by the shift of the specific ROS profile towards the right (Figure 2). The results conclusively demonstrated that hESC had the lowest innate basal intracellular levels of hydrogen peroxide and superoxide among the four different cell types examined. Hence, the observed higher resistance of undifferentiated hESC to oxidative stress and genotoxic stress compared to somatic cell types, as reported by our previous studies [5, 6], could be due to lower innate basal intracellular levels of ROS within hESCs compared to somatic cell types.

Our findings are consistent with previous studies which showed that ROS play crucial roles as secondary messengers during stem cell differentiation into various somatic lineages [10,11], so that an increase in ROS levels in differentiated stem cell progenies is therefore expected. Hirano and Tamae [12] and Kuboyama et al. [13], also reported that undifferentiated embryonic stem cells were more resistant to DNA damage induced by the ROS - 8-oxoguanine (which is known to generate GC-to-TA point mutations in genomic DNA), as compared to their differentiated progenies. These findings are thus consistent with the results of this study, as well as our previous studies $[5,6]$.

Future studies will be needed to investigate the mechanism by which hESCs maintains lower innate basal intracellular ROS levels compared to somatic cell types, e.g. due to higher levels of antioxidants and ROS protective enzymes such as superoxide dismutase.

\section{Acknowledgments}

This work was partially supported by grants R2210000 53515 and R221000085733 from the National University Health System (Singapore).

\section{References}

1. Guetens G, De Boeck G, Highley M, van Oosterom AT, de Bruijn EA. Oxidative DNA damage: biological significance and methods of analysis. Crit Rev Clin Lab Sci. 2002;39:331-457. doi: 10.1080/10408360290795547.

2. Loft S, Deng XS, Tuo J, Wellejus A, S rensen M, Poulsen HE. Experimental study of oxidative DNA damage. Free Radic Res. 1998;29:525-539. doi: 10.1080/10715769800300571.

3. Yin H, Casey PS, McCall MJ, Fenech M. Effects of surface chemistry on cytotoxicity, genotoxicity, and the generation of reactive oxygen species induced by $\mathrm{ZnO}$ nanoparticles. Langmuir. 2010;26:15399-15408. doi: 10.1021/la101033n.

4. Shukla RK, Sharma V, Pandey AK, Singh S, Sultana S, Dhawan A. ROS-mediated genotoxicity induced by titanium dioxide nanoparticles in human epidermal cells. Toxicol In Vitro. 2011;25:231-241. doi: 10.1016/j.tiv.2010.11.008.

5. George S, Heng BC, Vinoth KJ, Kishen A, Cao T. Comparison of the response of human embryonic stem cells and their 
differentiated progenies to oxidative stress. Photomed Laser Surg. 2009;27:669-674. doi: 10.1089/pho.2008.2354.

6. Vinoth KJ, Heng BC, Poonepalli A et al. Human embryonic stem cells may display higher resistance to genotoxic stress as compared to primary explanted somatic cells. Stem Cells Dev. 2008;17:599-607. doi: 10.1089/scd.2007.0088.

7. Collins AR. Investigating oxidative DNA damage and its repair using the comet assay. Mutat Res. 2009;681:24-32. doi: 10.1016/j.mrrev.2007.10.002.

8. Azqueta A, Shaposhnikov S, Collins AR. DNA oxidation: investigating its key role in environmental mutagenesis with the comet assay. Mutat Res. 2009;674:101-108. doi: 10.1016/j. mrgentox.2008.10.013.

9. Karanjawala ZE, Murphy N,Hinton DR, Hsieh CL,Lieber MR. Oxygen metabolism causes chromosome breaks and is associated with the neuronal apoptosis observed in DNA double- -strand break repair mutants. Curr Biol. 2002;12:397-402. doi: 10.1016/S0960-9822(02)00684-X.

10. Ji AR, Ku SY, Cho MS et al. Reactive oxygen species enhance differentiation of human embryonic stem cells into mesendodermal lineage. Exp Mol Med. 2010;42:175-186. doi: 10.3858/emm.2010.42.3.018.

11. Sart S, Song L, Li Y. Controlling redox status for stem cell survival, expansion, and differentiation. Oxidative Medicine and Cellular Longevity. 2015. doi: 10.1155/2015/105135.

12. Hirano T, Tamae K. Differentiation of embryonic stem cells and oxidative DNA damage/DNA repair systems. J Stem Cell Res Ther. 2012;S10:005. doi: 10.4172/2157-7633.S10-005.

13. Kuboyama A, Tanaka S, Kawai K, Kasai H, Morii H. 8-Hydroxyguanine levels and repair capacity during mouse embryonic stem cell differentiation. Free Radic Res. 2011;45:527-533. doi: 10.3109/10715762.2011.555481.

Submitted: 26 May, 2015

Accepted after reviews: 7 July, 2015

Available as AoP: 8 July, 2015 